\title{
Evaluation of positive ductal margins of biliary tract cancer in intraoperative histological examination
}

\author{
JUNJI UEDA ${ }^{1,2}$, HIROSHI YOSHIDA ${ }^{2}$, YASUHIRO MAMADA ${ }^{1}$, NOBUHIKO TANIAI ${ }^{1}$, MASATO YOSHIOKA $^{1}$, \\ ATSUSHI HIRAKATA ${ }^{2}$, YOUICHI KAWANO ${ }^{1}$, YOSHIAKI MIZUGUCHI ${ }^{1}$, TETSUYA SHIMIZU ${ }^{1}$, \\ TOMOHIRO KANDA ${ }^{1}$, HIDEYUKI TAKATA ${ }^{2}$, RYOTA KONDO $^{1}$ and EIJI UCHIDA ${ }^{1}$ \\ ${ }^{1}$ Department of Surgery, Nippon Medical School, Bunkyo-ku, Sendagi, Tokyo 113-8603; \\ ${ }^{2}$ Department of Surgery, Nippon Medical School Tamanagayama Hospital, Tokyo 206-8512, Japan
}

Received October 22, 2017; Accepted September 13, 2018

DOI: $10.3892 / \mathrm{ol} .2018 .9479$

\begin{abstract}
At present the only method available to confirm microscopic infiltration of cancer into ductal margins during surgery, is intraoperative histological examination. In the present study, the status of the surgical margins and postoperative course were evaluated to determine any correlation between remnant carcinoma and postoperative survival. All consecutive patients who underwent resection for biliary tract cancer between January 2004 and May 2012 were identified from a database. Positive margin cases were divided into two groups, invasive carcinoma and carcinoma in situ (CIS). Immunohistochemical staining targeting Ki67 and p53 for positive margins was performed. Cases of major vessel invasion were significantly increased in the positive group compared with the negative group. The recurrence rate was significantly lower in the CIS group compared with the invasive group. The survival rate was significantly increased in the CIS group compared with the invasive group. The expression levels of p53 and Ki67 were significantly increased in the invasive group compared with the CIS group. No statistical correlations were observed between the expression of p53 or Ki67 and the survival or recurrence of disease. In the positive group, resected margin status was the principal factor associated with recurrence-free survival according to Cox-regression analysis. In conclusion, the status of the resected margins in the positive group was the most important factor for postoperative survival and recurrence in cholangiocarcinoma, not immunohistochemical staining targeting Ki67 and p53.
\end{abstract}

Correspondence to: Dr Junji Ueda, Department of Surgery, Nippon Medical School Tamanagayama Hospital, 1-7-1 Nagayama, Tama, Tokyo 206-8512, Japan

E-mail: junji0821@nms.ac.jp

Key words: cholangiocarcinoma, intraoperative histological examination, surgery

\section{Introduction}

Biliary tract cancer, including cancers of the gallbladder and extrahepatic bile duct, is rare but highly fatal (1), and complete surgical resection is the only treatment that offers a chance of cure in patients (2). Although preoperative diagnosis has been improved by modern imaging techniques, it is still difficult to diagnose the extent of cholangiocarcinoma infiltration (3). Currently, the only method to confirm microscopic infiltration of cancer into ductal resection margins during surgery is intraoperative histological examination. However, some reports have revealed that prognosis for patients with positive bile duct stumps does not differ significantly from that of negative patients (4). Moreover, recent studies have shown that additional resection of a positive proximal bile duct margin does not offer any survival advantage $(4,5)$. It remains controversial whether additional resection of the bile duct has a favorable impact on the patient outcomes (5). The impact of remnant carcinoma in situ (CIS) at the bile duct stump on on postoperative course remain unclear (4). In this study, surgical margin status and postoperative course were evaluated in 93 patients with biliary tract cancer to determine any correlation between remnant carcinoma and postoperative survival. Positive margin cases were divided into two groups, invasive carcinoma and carcinoma in situ, and the postoperative survival and recurrence-free survival were evaluated. Immunohistochemical staining targeting Ki67 and p53 for positive margins and invasion portion was conducted to evaluate the correlation between expression of these proteins and prognosis.

\section{Patients and methods}

Patients. All consecutive patients who underwent resection for biliary tract cancer at our institution between January 2004 and May 2012 were identified from a database. The number of cases was 93. Patients with carcinoma of the ampulla of Vater and intrahepatic cholangiocarcinoma were excluded. Data collection and analysis were performed according to institution guidelines in conformance with the ethical standards of the Declaration of Helsinki. The present study was approved by the Ethics Committee of Nippon Medical School Tamanagayama Hospital 
Table I. Clinical and pathological characteristics of the study.

\section{Characteristics}

Age (years)

Sex

Male/female

Disease

CBD/HCCA/GB

Ductal margin

Positive/negative

pT

$1 / 2 / 3 / 4$

$\mathrm{pN}$

$0 / 1 / 2 / 3$

$\mathrm{pM}$

0/1

$\mathrm{V}$

$0 / 1 / 2 / 3$

ly

$0 / 1 / 2 / 3$

ne

$0 / 1 / 2 / 3$

Hinf

$0 / 1 / 2 / 3$

$\mathrm{Du}$

$0 / 1 / 2 / 3$

Panc

$0 / 1 / 2 / 3$

PA

0/1/2/3

$\mathrm{PV}$

$0 / 1 / 2 / 3$

$\mathrm{pEM}$

0/1/2

$\mathrm{pHM}$

$0 / 1 / 2$

pDM

$0 / 1 / 2$

Cur

$\mathrm{A} / \mathrm{B} / \mathrm{C}$

pStage

I/II/III/IVa/IVb
Table II. Clinicopathological findings in the positive and negative groups.

\begin{tabular}{|c|c|c|c|}
\hline Variable & $\begin{array}{c}\text { Negative } \\
(n=73)\end{array}$ & $\begin{array}{c}\text { Positive } \\
(\mathrm{n}=20)\end{array}$ & P-value \\
\hline Age (years) & 68.9 & 69.1 & 0.9416 \\
\hline \multicolumn{4}{|l|}{ Sex } \\
\hline Male/female & $45 / 28$ & $16 / 4$ & 0.1258 \\
\hline \multicolumn{4}{|l|}{ Disease } \\
\hline $\mathrm{CBD} / \mathrm{HCCA} / \mathrm{GB}$ & $35 / 19 / 19$ & $12 / 5 / 3$ & 0.7102 \\
\hline \multicolumn{4}{|l|}{$\mathrm{pT}$} \\
\hline $1 / 2 / 3 / 4$ & $6 / 16 / 24 / 27$ & $5 / 5 / 7 / 3$ & 0.1033 \\
\hline \multicolumn{4}{|l|}{$\mathrm{pN}$} \\
\hline $0 / 1 / 2 / 3$ & $40 / 18 / 12 / 3$ & $10 / 5 / 5 / 0$ & 0.6260 \\
\hline \multicolumn{4}{|l|}{$\mathrm{pM}$} \\
\hline $0 / 1$ & $73 / 0$ & $19 / 1$ & 0.0548 \\
\hline \multicolumn{4}{|l|}{$\mathrm{v}$} \\
\hline $0 / 1 / 2 / 3$ & $25 / 16 / 23 / 4$ & $5 / 5 / 7 / 1$ & 0.9096 \\
\hline \multicolumn{4}{|l|}{ ly } \\
\hline $0 / 1 / 2 / 3$ & $12 / 22 / 25 / 12$ & $2 / 9 / 6 / 2$ & 0.5706 \\
\hline \multicolumn{4}{|l|}{ ne } \\
\hline $0 / 1 / 2 / 3$ & $19 / 9 / 14 / 25$ & $3 / 2 / 5 / 9$ & 0.6623 \\
\hline \multicolumn{4}{|l|}{ Hinf } \\
\hline \multicolumn{4}{|l|}{$\mathrm{Du}$} \\
\hline $0 / 1 / 2 / 3$ & $43 / 0 / 7 / 3$ & 13/1/1/0 & 0.1775 \\
\hline \multicolumn{4}{|l|}{ Panc } \\
\hline $0 / 1 / 2 / 3$ & $30 / 10 / 7 / 6$ & $10 / 4 / 0 / 0$ & 0.2269 \\
\hline \multicolumn{4}{|l|}{ PA } \\
\hline $0 / 1 / 2 / 3$ & $65 / 1 / 0 / 0$ & $12 / 5 / 1 / 0$ & 0.0218 \\
\hline \multicolumn{4}{|l|}{ PV } \\
\hline $0 / 1 / 2 / 3$ & $59 / 2 / 2 / 2$ & $15 / 2 / 0 / 1$ & 0.4286 \\
\hline \multicolumn{4}{|l|}{$\mathrm{pEM}$} \\
\hline $0 / 1 / 2$ & $51 / 8 / 9$ & $9 / 6 / 4$ & 0.0530 \\
\hline \multicolumn{4}{|l|}{ pStage } \\
\hline I/II/III/IVa/IVb & $4 / 13 / 22 / 28 / 6$ & $2 / 5 / 6 / 6 / 1$ & 0.8305 \\
\hline
\end{tabular}

CBD, common bile duct cancer; HCCA, hiller cholangiocarcinoma; GB, gall bladder cancer; $\mathrm{pT}$, pathological depth of invasion; $\mathrm{pN}$, pathological lymph node metastasis category; $v$, minor vein invasion; ly, lymph duct invasion; ne, neural invasion; Hinf, hepatic invasion; Du, duodenal invasion; Panc, pancreas invasion; pEM, patholoical dissected margin; PA, pulmonary artery; PV, pulmonary vein.

Margin status. In practice, margins of at least $5 \mathrm{~mm}$ are recommended to achieve curative resection. Intraoperative histological examination is routinely used to assess the bile duct margin. If evidence or possibility of a tumour-positive margin is observed during this examination, additional resection of the proximal duct is performed, but only as far as it is technically feasible. All duct margins submitted for intraoperative histological examination are then re-evaluated later by permanent pathology. Margin status is classified as negative, positive with CIS, or positive with invasive carcinoma. start of the study. 
A

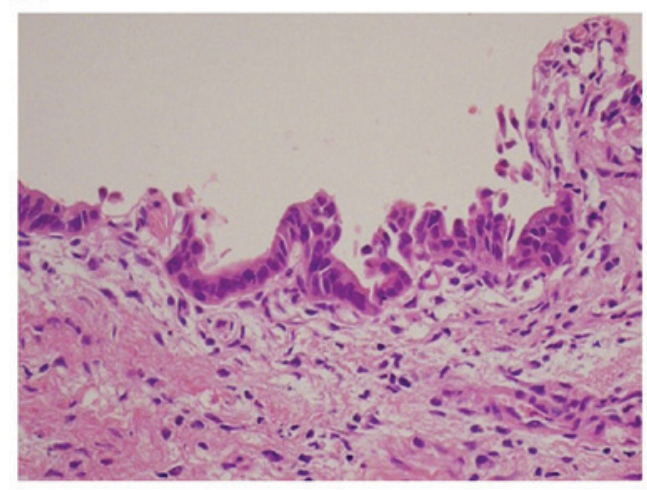

B

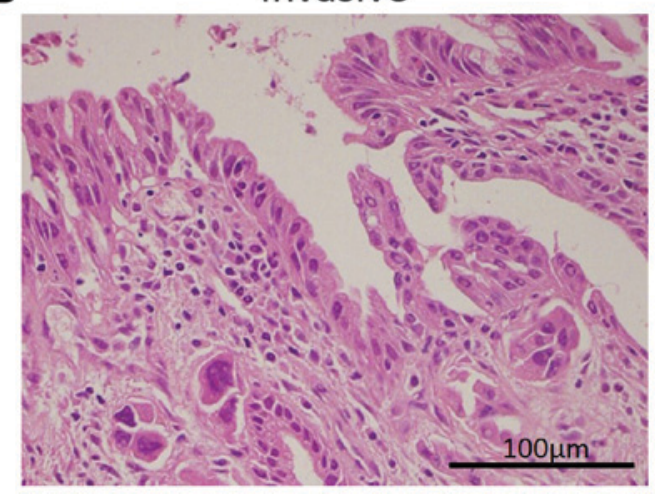

Figure 1. Status of the resected margin in the positive group. (A) CIS at the bile duct stump and (B) invasive carcinoma at the surgical margin. Magnification, x600. CIS, carcinoma in situ.

Immunostaining and scoring. In this study we used the cancer tissues in ductal margin. The paraffin-embedded serial tissue sections (3.5- $\mu \mathrm{m}$ thick) were subjected to immunostaining using Histofine Simple Stain Max-PO kits. Antigen Activation Liquid (pH 9.0; Nichirei Biosciences, Inc., Tokyo, Japan) at $121^{\circ} \mathrm{C}$ for $15 \mathrm{~min}$. Endogenous peroxidase was blocked in $0.3 \%$ hydrogen peroxide and methanol for $30 \mathrm{~min}$. Sections were then incubated with antibodies for p53 (cat. no. 20050705; dilution, 1:50) and Ki-67 (MIB1; cat. no. 20049476; dilution, 1:500; both Dako; Agilent Technologies, Inc., Santa Clara, CA, USA) in phosphate-buffered saline containing $1 \%$ bovine serum albumin (Sigma-Aldrich; Merck KGaA, Darmstadt, Germany) for $16 \mathrm{~h}$ at $4^{\circ} \mathrm{C}$. The sections were further incubated with the Histofine Simple Stain ${ }^{\mathrm{TM}}$ MAX-PO (R; Nichirei Biosciences, Inc.) for $30 \mathrm{~min}$, and peroxidase activity was visualized by 3,3'-diaminobenzidine. The sections were then counterstained with hematoxylin. Evaluation of the degree and positive ratio of p53 and ki-67 immunostaining was conducted using the following scale: 0 , no staining; $1+$, mild staining; $2+$, moderate staining; and $3+$, intense staining; and 0 , no staining; $1+, 1-30 \%$ positive ratio in cancer cells; $2+, 31-60 \%$; and $3+,>60 \%(6)$.

Clinicopathological features. Clinicopathological data included age, sex, location of the main tumour, surgical dissection margin, pathological depth of invasion (pT), major vessel invasion (A, PV), minor vein invasion (v), lymph duct invasion (ly), pathological lymph node metastasis category $(\mathrm{pN})$, pancreas invasion (Panc), duodenal invasion (Du), hepatic invasion (Hinf), neural invasion (ne), bile duct distal margin (pDM), horizon margin ( $\mathrm{pHE})$, patholoical dissected margin ( $\mathrm{pEM}$ ), stage characterized according to the Japanese Society of Biliary Surgery Classification (7), date of recurrence, recurrence site, and date and cause of death. Sites of disease recurrence were confirmed by computed tomography and/or magnetic resonance imaging and/or positron emission tomography/computed tomography. When new findings of suspected cancer appeared, and cancer progression was observed by serial imaging, radiologic evidence of tumour recurrence was accepted without a biopsy. The date of the first radiologic finding of suspected cancer was recorded as the date of initial disease recurrence.
Statistical analysis. Statistical analysis was performed using the statistical software package SPSS, version 16.0 (SPSS, Chicago, IL, USA). Categorical variables were compared using the $\chi^{2}$ test or Fisher's exact test. Survival was calculated using the Kaplan-Meier method and compared by the log-rank test and Cox-regression analysis. $\mathrm{P}<0.05$ was considered to indicate a statisticaly significant difference.

\section{Results}

During the study, 93 patients underwent curative intent resection. The clinical and pathological characteristics of the study participants are shown in Table I. The mean age of the patients was 69 years. The age range was 35-85 years and 61 patients were male $(65.6 \%)$ and 32 patients were female $(34.4 \%)$. There were 20 cases $(21.5 \%)$ of positive-margin intraoperative histological examination and permanent pathological findings. The distribution of disease was as follows: 46 cases $(49.5 \%)$ of common bile duct cancer (CBD), 25 cases (26.9\%) of hiller cholangiocarcinoma (HCCA), and 22 cases (23.6\%) of gall bladder cancer (GB). Operative procedures included 34 cases of pancreaticoduodenectomy, 32 cases of hemihepatectomy with bile duct resection, 16 cases of partial hepatectomy with bile duct resection, 8 cases cholecystectomy with bile duct resection, and 3 cases of hepatopancreatoduodenectomy. Next, patients were divided into two groups, a positive margin group (positive) and a negative margin group (negative). Clinicopathological findings were comparatively evaluated between the positive and negative groups (Table II). Cases with major vessel invasion were significantly higher in the positive group than in the negative group $(\mathrm{P}=0.0218)$. We focused on the positive group and further divided these patients into two subgroups according to resected margin status: CIS at the bile duct stump (CIS group, n=8) (Fig. 1A) and invasive carcinoma at any surgical margin (Invasive group, $n=12$ ) (Fig. 1B). No significant difference in clinicopathological findings between the two groups was observed. Recurrence and survival were significantly lower and higher, respectively, in the CIS group than in the Invasive group (Fig. 2). Immunohistochemical staining targeting p53 and Ki67 was conducted in the positive margins (Fig. 3). The expression levels of p53 and Ki67 in ductal margins were significantly higher in the Invasive group 

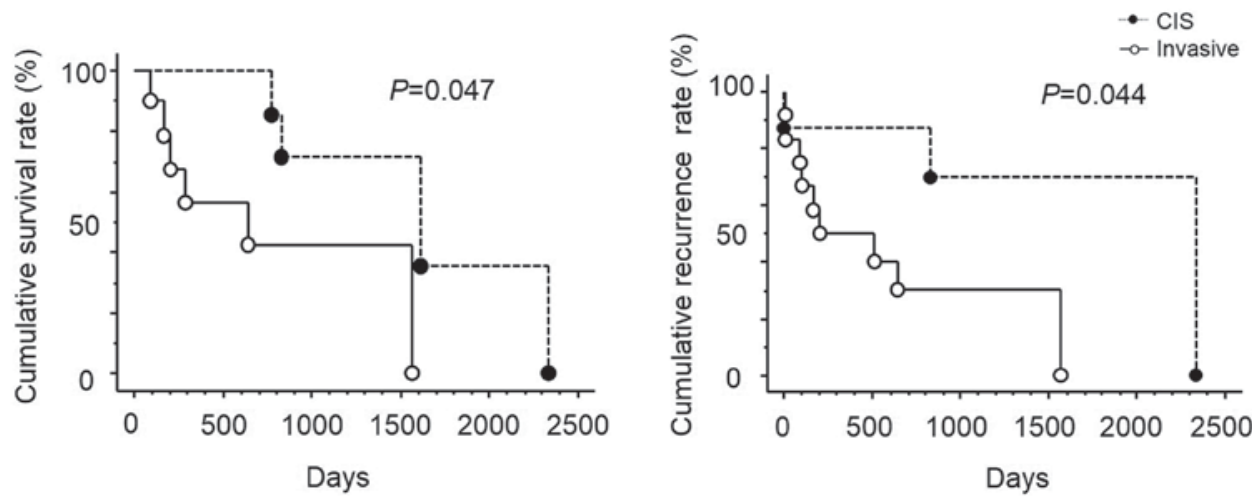

Figure 2. Recurrence and survival were significantly lower and higher, respectively, in the CIS group compared with the Invasive group. CIS, carcinoma in situ.
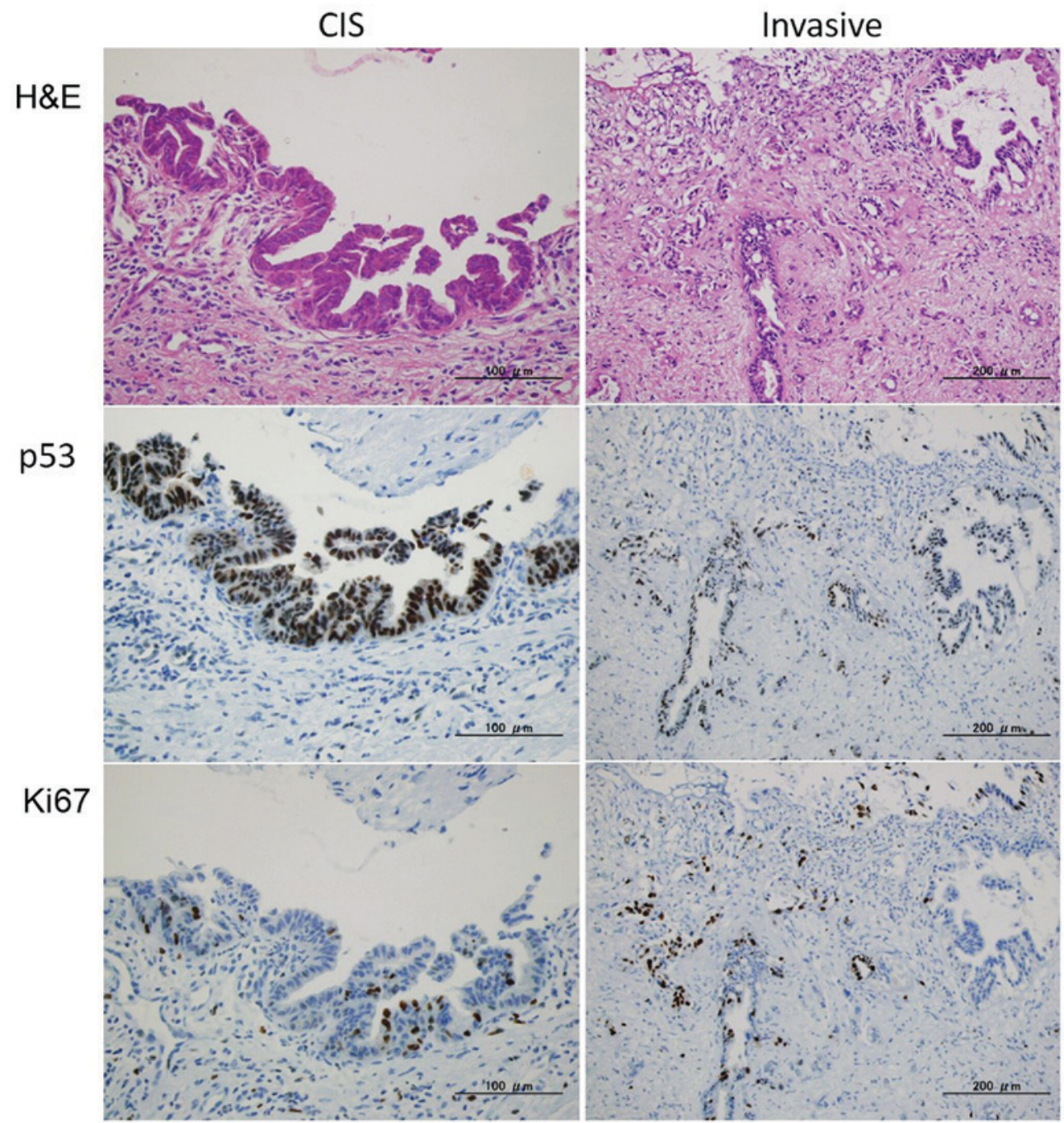

Figure 3. Immunohistochemical staining targeting p53 and Ki67 in the positive margin group. Magnification, left row x600; right row, x400. CIS, carcinoma in situ.

$(\mathrm{P}=0.035)$ (Table III). However, the expression levels in the invasion portion were not significantly different between the two groups. Moreover, no statistical correlations between the expression levels of p53 and Ki67 and survival and recurrence were observed (Fig. 4). In the positive group, resected margin status was the most important factor for recurrence-free survival according to Cox-regression analysis (Table IV).

\section{Discussion}

Although histologically negative margins have been recognized as the most important independent determinant of survival, the additional resection of a diagnosed positive proximal bile duct is still controversial (4). Many studies have reported positive surgical margins as an important predictor of poor 
Table III. No statistical correlation between the clinicopathological findings and expression of p53 and Ki67 was observed in the CIS and invasive groups.

\begin{tabular}{|c|c|c|c|}
\hline & $\begin{array}{c}\text { CIS } \\
(n=8)\end{array}$ & $\begin{array}{l}\text { Invasive } \\
(\mathrm{n}=12)\end{array}$ & P-value \\
\hline Age (years) & 65.6 & 71.4 & 0.1462 \\
\hline \multicolumn{4}{|l|}{ Sex } \\
\hline Male/female & $5 / 3$ & $11 / 1$ & 0.1101 \\
\hline \multicolumn{4}{|l|}{ Disease } \\
\hline CBD/HCCA/GB & $5 / 2 / 1$ & $6 / 4 / 2$ & 0.8594 \\
\hline \multicolumn{4}{|l|}{$\mathrm{pT}$} \\
\hline $1 / 2 / 3 / 4$ & $2 / 1 / 3 / 2$ & $3 / 4 / 4 / 1$ & 0.6276 \\
\hline \multicolumn{4}{|l|}{$\mathrm{pN}$} \\
\hline $0 / 1 / 2 / 3$ & $6 / 1 / 1 / 0$ & $4 / 4 / 4 / 0$ & 0.1889 \\
\hline \multicolumn{4}{|l|}{$\mathrm{pM}$} \\
\hline $0 / 1$ & $7 / 1$ & $12 / 0$ & 0.2089 \\
\hline \multicolumn{4}{|l|}{$\mathrm{v}$} \\
\hline $0 / 1 / 2 / 3$ & $2 / 2 / 4 / 0$ & $5 / 3 / 2 / 1$ & 0.5924 \\
\hline \multicolumn{4}{|l|}{ ly } \\
\hline $0 / 1 / 2 / 3$ & $1 / 3 / 3 / 1$ & $2 / 6 / 3 / 1$ & 0.9065 \\
\hline \multicolumn{4}{|l|}{ ne } \\
\hline $0 / 1 / 2 / 3$ & $2 / 2 / 1 / 3$ & $2 / 0 / 4 / 6$ & 0.2440 \\
\hline \multicolumn{4}{|l|}{ Hinf } \\
\hline $0 / 1 / 2 / 3$ & $5 / 1 / 1 / 1$ & $8 / 3 / 0 / 1$ & 0.5784 \\
\hline \multicolumn{4}{|l|}{$\mathrm{Du}$} \\
\hline $0 / 1 / 2 / 3$ & $8 / 0 / 0 / 0$ & $11 / 0 / 1 / 0$ & 0.4022 \\
\hline \multicolumn{4}{|l|}{ Panc } \\
\hline $0 / 1 / 2 / 3$ & $6 / 2 / 0 / 0$ & $10 / 2 / 0 / 0$ & 0.6481 \\
\hline \multicolumn{4}{|l|}{ PA } \\
\hline $0 / 1 / 2 / 3$ & $6 / 2 / 0 / 0$ & $11 / 0 / 1 / 0$ & 0.1478 \\
\hline \multicolumn{4}{|l|}{ PV } \\
\hline $0 / 1 / 2 / 3$ & $5 / 2 / 0 / 1$ & $11 / 0 / 0 / 0$ & 0.0709 \\
\hline \multicolumn{4}{|l|}{$\mathrm{pEM}$} \\
\hline $0 / 1 / 2$ & $5 / 2 / 1$ & $5 / 4 / 3$ & 0.6360 \\
\hline \multicolumn{4}{|l|}{ pStage } \\
\hline I/II/III/IVa/IVb & $0 / 2 / 2 / 3 / 1$ & $2 / 3 / 4 / 3 / 0$ & 0.5112 \\
\hline \multicolumn{4}{|l|}{ p53 } \\
\hline Negative/positive & $4 / 4$ & $1 / 11$ & 0.0350 \\
\hline \multicolumn{4}{|l|}{ Ki67 } \\
\hline$<50 \% />50 \%$ & $4 / 4$ & $1 / 11$ & 0.0350 \\
\hline
\end{tabular}

CIS, carcinoma in situ; CBD, common bile duct cancer; HCCA, hiller cholangiocarcinoma; GB, gall bladder cancer; pT, pathological depth of invasion; $\mathrm{pN}$, pathological lymph node metastasis category; $\mathrm{pN}$, pathological lymph node metastasis category; $\mathrm{v}$, minor vein invasion; ly, lymph duct invasion; ne, neural invasion; Hinf, hepatic invasion; Du, duodenal invasion; Panc, pancreas invasion; $p E M$, patholoical dissected margin; PA, pulmonary artery; PV, pulmonary vein.

prognosis (8-11). According to several reports, however, the prognosis for patients with positive bile duct stumps does not
Table IV. Cox-regression analysis of recurrence-free survival in the positive group.

\begin{tabular}{lcrc}
\hline & \multicolumn{3}{c}{ Hazard } \\
Recurrence-free survival & P-value & ratio & $95 \%$ CI \\
\hline Ductal margin & 0.0229 & 15.8745 & $1.468-171.7$ \\
p53 & 0.0778 & 0.0662 & $0.003-1.354$ \\
Ki67 & 0.4885 & 2.6464 & $0.1686-41.55$ \\
\hline
\end{tabular}

CI, confidence interval.

differ significantly from that of carcinoma-negative patients. Moreover, some studies have even described long-term survival for patients with a positive surgical bile duct stump $(12,13)$. Histologically positive surgical bile duct stumps can be further subclassified into two subtypes: Invasive carcinoma and CIS (4). Some reports have shown the survival rate of CIS groups to be no less than that of margin negative groups and higher than that of invasive groups (4). In this study, bile duct stumps were also divided into the same two groups. The recurrence and survival rates in the CIS group were not lower than those in the negative group (data not shown). The recurrence and survival rates were significantly lower and higher in the CIS group than in the Invasive group. The biological nature of main tumours with extensive superficial spread, which is likely to be responsible for remnant CIS, tends to be less malignant than that of conventional cholangiocarcinoma (4). Main tumours of extrahepatic bile duct carcinoma with extensive superficial spreading tend to have shallower invasion, more localized type gross appearance, and a more defined histological differentiation than conventional cholangiocarcinoma (14-16). Despite the usefulness of intraoperative histological examination in assessing tumour involvement in the bile duct, there are limitations to this method $(17,18)$. A common feature of tumour spread is a submucosal pattern extending from 1 to $2 \mathrm{~cm}$ beyond the abnormal lesions, as observed in an imaging study (19). Intraoperative examination of margin status can be difficult due to the biological characteristics of this tumour, which involve the proximal microscopic spread of the disease along the bile duct, extending beyond the palpable macroscopic boundaries $(17,20)$. Intraoperative histological examination may inaccurately differentiate between invasive carcinoma from epithelial atypia or dysplasia, especially in cases of inflammation of the ducts due to obstruction or a biliary drainage procedure (21). These factors lead to inaccurate diagnosis. Moreover, in our study, major vessel invasion was significantly more frequent in the carcinoma-positive group than in the carcinoma-negative group. When a positive surgical margin is identified, the state of the positive surgical margin must be examined more closely. The bile duct stump was also examined using immunohistochemical staining targeting $\mathrm{Ki} 67$ and p53. Ki67 is a prognostic marker of tumour proliferation that has been extensively researched in retrospective studies (22). Its cellular location is strongly cell cycle dependent $(23,24)$. Ki67 levels are low during G1 and early $\mathrm{S}$ phase and peak during mitosis (22). p53 functions as a transcription factor involved in cell-cycle control, DNA repair, 



Figure 4. No statistical correlation was observed between the expression of p53 and Ki67 in the positive margin, survival and recurrence rates.

apoptosis and cellular stress responses (25). The tumour suppressor gene p53 was the first identified cancer gene (26). During cell cycle arrest, p53 functions by upregulating cyclin-dependent kinase (cdk) inhibitor p21, which can be detected as an immunohistochemical overexpression (27-29). Inactivation of $\mathrm{p} 53$ caused by missense mutations or interaction with oncogenic viral proteins results in a selective growth advantage for cancer cells $(30,31)$. Most p53 gene mutations stop tumour suppressor activity (32). The frequency of p53 overexpression in cholangiocarcinoma is reported to vary from 19 to $58 \%$ (32-37). In this study, immunohistochemical staining targeting Ki67 and p53 in surgical bile duct stumps did not show a correlation with overall survival or recurrence-free survival. The histopathological findings of surgical bile duct stumps, whether positive with CIS or positive with invasive carcinoma, were more important than immunohistochemical staining findings.

In this study, we did not perform the experiment using the cell lines. Further studies were recommended to verify our results.

Some report showed the radiotherapy for biliary tract cancer (38). However, the radiotherapy for biliary tract cancer is not established. Some report showed the benefit of disease-free survival (39), but the evidence is insufficient due to the small number $(<10)$ of 5-year survivors and retrospective study (38). Moreover, some reports have revealed the effectiveness of chemotherapy for biliary tract cancer (40). However, adjuvant chemotherapy for biliary tract cancer has not been investigated. Some reports evaluating the impact of adjuvant treatment with systemic chemotherapy suggested a benefit for high-risk patients with positive lymph nodes or positive resection margins $(41,42)$. Prospective studies are needed. A large multi-national study (ACTICCA-1) is evaluating the combination of gemcitabine plus cisplatin for adjuvant chemotherapy with results expected within the next years $(40,43)$.

Intraoperative histological examination of the surgical margin of the bile duct is essential during biliary tract cancer surgery. The status of the resected margin in the positive group was the most important factor for postoperative survival and recurrence in biliary tract cancer.

\section{Acknowledgements}

Not applicable.

\section{Funding}

No funding was received.

\section{Availability of data and materials}

The datasets used and/or analyzed during the current study are available from the corresponding author on reasonable request.

\section{Authors' contributions}

JU, HY and EU designed the study, performed the experiments and wrote the manuscript. YMa, NT, MY, AH, YK, YMi, TS, TK, HT and RK performed the experiments. All authors read and approved the final manuscript.

\section{Ethics approval and consent to participate}

The present study was approved by the Ethics committee of Nippon Medical School, Tamanagayama Hospital 
(approval no. 407) and written informed consent was obtained from all participants.

\section{Patient consent for publication}

Not applicable.

\section{Competing interests}

The authors declare that they have no competing interests.

\section{References}

1. Castro FA, Koshiol J, Hsing AW and Devesa SS: Biliary tract cancer incidence in the United States-demographic and temporal variations by anatomic site. Int J Cancer 133: 1664-1671, 2013.

2. Konishi M, Ochiai A, Ojima H, Hasebe T, Mano M, Ohta T, Ito I, Sasaki K, Yasukawa S, Shimada K, et al: A new histological classification for intra-operative histological examination of the ductal resection margin in cholangiocarcinoma. Cancer Sci 100: 255-260, 2009.

3. Konishi M, Iwasaki M, Ochiai A, Hasebe T, Ojima H and Yanagisawa A: Clinical impact of intraoperative histological examination of the ductal resection margin in extrahepatic cholangiocarcinoma. Br J Surg 97: 1363-1368, 2010.

4. Nakanishi Y, Kondo S, Zen Y, Yonemori A, Kubota K, Kawakami H, Tanaka E, Hirano S, Itoh T and Nakanuma Y: Impact of residual in situ carcinoma on postoperative survival in 125 patients with extrahepatic bile duct carcinoma. J Hepatobiliary Pancreat Sci 17: 166-173, 2010.

5. Ribero D, Amisano M, Lo Tesoriere R, Rosso S, Ferrero A and Capussotti L: Additional resection of an intraoperative margin-positive proximal bile duct improves survival in patients with hilar cholangiocarcinoma. Ann Surg 254: 776-783, 2011

6. Matsuda Y, Yamamoto T, Kudo M, Kawahara K, Kawamoto M, Nakajima Y, Koizumi K, Nakazawa N, Ishiwata T and Naito Z: Expression and roles of lumican in lung adenocarcinoma and squamous cell carcinoma. Int J Oncol 33: 1177-1185, 2008.

7. Miyazaki M, Ohtsuka M, Miyakawa S, Nagino M, Yamamoto M, Kokudo N, Sano K, Endo I, Unno M, Chijiiwa K, et al: Classification of biliary tract cancers established by the Japanese Society of Hepato-Biliary-Pancreatic Surgery: 3(rd) English edition. J Hepatobiliary Pancreat Sci 22: 181-196, 2015 .

8. Kayahara M, Nagakawa T, Ohta T, Kitagawa H, Tajima H and Miwa K: Role of nodal involvement and the periductal soft-tissue margin in middle and distal bile duct cancer. Ann Surg 229: 76-83, 1999.

9. Burke EC, Jarnagin WR, Hochwald SN, Pisters PW, Fong Y and Blumgart LH: Hilar Cholangiocarcinoma: Patterns of spread, the importance of hepatic resection for curative operation and a presurgical clinical staging system. Ann Surg 228 : 385-394, 1998

10. DeOliveira ML, Cunningham SC, Cameron JL, Kamangar F, Winter JM, Lillemoe KD, Choti MA, Yeo CJ and Schulick RD: Cholangiocarcinoma: Thirty-one-year experience with 564 patients at a single institution. Ann Surg 245: 755-762, 2007.

11. Kondo S, Takada T, Miyazaki M, Miyakawa S, Tsukada K, Nagino M, Furuse J, Saito H, Tsuyuguchi T, Yamamoto M, et al: Guidelines for the management of biliary tract and ampullary carcinomas: Surgical treatment. J Hepatobiliary Pancreat Surg 15: 41-54, 2008.

12. Bhuiya MR, Nimura Y, Kamiya J, Kondo S, Nagino M and Hayakawa N: Clinicopathologic factors influencing survival of patients with bile duct carcinoma: Multivariate statistical analysis. World J Surg 17: 653-657, 1993.

13. Jarnagin WR, Fong Y, DeMatteo RP, Gonen M, Burke EC, Bodniewicz BS J, Youssef BA M, Klimstra D and Blumgart LH: Staging, resectability, and outcome in 225 patients with hilar cholangiocarcinoma. Ann Surg 234: 507-519, 2001.

14. Igami T, Nagino M, Oda K, Nishio H, Ebata T, Yokoyama Y and Shimoyama Y: Clinicopathologic study of cholangiocarcinoma with superficial spread. Ann Surg 249: 296-302, 2009.
15. Nakanishi Y,Zen Y, Kawakami H, Kubota K, Itoh T, Hirano S, Tanaka E, Nakanuma Y and Kondo S: Extrahepatic bile duct carcinoma with extensive intraepithelial spread: A clinicopathological study of 21 cases. Mod Pathol 21: 807-816, 2008.

16. Ojima H, Kanai Y, Iwasaki M, Hiraoka N, Shimada K, Sano T, Sakamoto Y, Esaki M, Kosuge T, Sakamoto M and Hirohashi S: Intraductal carcinoma component as a favorable prognostic factor in biliary tract carcinoma. Cancer Sci 100: 62-70, 2009.

17. Endo I, House MG, Klimstra DS, Gonen M, D'Angelica M, Dematteo RP, Fong Y, Blumgart LH and Jarnagin WR: Clinical significance of intraoperative bile duct margin assessment for hilar cholangiocarcinoma. Ann Surg Oncol 15: 2104-2112, 2008.

18. Okazaki Y, Horimi T, Kotaka M, Morita S and Takasaki M: Study of the intrahepatic surgical margin of hilar bile duct carcinoma. Hepatogastroenterology 49: 625-627, 2002.

19. Baton O, Azoulay D, Adam DV and Castaing D: Major hepatectomy for hilar cholangiocarcinoma type 3 and 4: Prognostic factors and longterm outcomes. J Am Coll Surg 204: 250-260, 2007.

20. Sakamoto E, Nimura Y, Hayakawa N, Kamiya J, Kondo S, Nagino M, Kanai M, Miyachi M and Uesaka K: The pattern of infiltration at the proximal border of hilar bile duct carcinoma: A histologic analysis of 62 resected cases. Ann Surg 227: 405-411, 1998.

21. Lee JH, Hwang DW, Lee SY, Park KM and Lee YJ: The proximal margin of resected hilar cholangiocarcinoma: The effect of microscopic positive margin on long-term survival. Am Surg 78: 471-477, 2012

22. Konstantinos K, Marios S, Anna M, Nikolaos K, Efstratios P and Paulina A: Expression of Ki-67 as proliferation biomarker in imprint smears of endometrial carcinoma. Diagn Cytopathol 41: 212-217, 2013.

23. IsolaJ,HelinH and KallioniemiOP:Immunoelectron-microscopic localization of a proliferation-associated antigen Ki-67 in MCF-7 cells. Histochem J 22: 498-506, 1990.

24. Verheijen R, Kuijpers HJ, Schlingemann RO, Boehmer AL, van Driel R, Brakenhoff GJ and Ramaekers FC: Ki-67 detects a nuclear matrix-associated proliferation-related antigen. I. Intracellular localization during interphase. J Cell Sci 92: 123-130, 1989.

25. Rufini A, Tucci P, Celardo I and Melino G: Senescence and aging: The critical roles of p53. Oncogene 32: 5129-5143, 2013.

26. Basu $A$ and Haldar S: The relationship between BcI2, Bax and p53: Consequences for cell cycle progression and cell death. Mol Hum Reprod 4: 1099-1109, 1998.

27. Greenblatt MS, Bennett WP, Hollstein $M$ and Harris CC: Mutations in the p53 tumor suppressor gene: Clues to cancer etiology and molecular pathogenesis. Cancer Res 54: 4855-4878, 1994.

28. Hussain SP and Harris CC: Molecular epidemiology of human cancer: Contribution of mutation spectra studies of tumor suppressor genes. Cancer Res 58: 4023-4037, 1998.

29. Kang YK, Kim WH and Jang JJ: Expression of G1-S modulators (p53, p16, p27, cyclin D1, Rb) and Smad4/Dpc4 in intrahepatic cholangiocarcinoma. Hum Pathol 33: 877-883, 2002.

30. Harris CC: Structure and function of the p53 tumor suppressor gene: Clues for rational cancer therapeutic strategies. J Natl Cancer Inst 88: 1442-1455, 1996.

31. Levine AJ: The p53 tumor-suppressor gene. N Engl J Med 326: 1350-1352, 1992.

32. Diamantis I, Karamitopoulou E, Perentes E and Zimmermann A: p53 protein immunoreactivity in extrahepatic bile duct and gallbladder cancer: Correlation with tumor grade and survival. Hepatology 22: 774-779, 1995.

33. Jan YY, Yeh TS, Yeh JN, Yang HR and Chen MF: Expression of epidermal growth factor receptor, apomucins, matrix metalloproteinases, and p53 in rat and human cholangiocarcinoma: Appraisal of an animal model of cholangiocarcinoma. Ann Surg 240: 89-94, 2004.

34. Ohashi K, Nakajima Y, Kanehiro H, Tsutsumi M, Taki J, Aomatsu Y, Yoshimura A, Ko S, Kin T, Yagura K, et al: Ki-ras mutations and p53 protein expressions in intrahepatic cholangiocarcinomas: relation to gross tumor morphology. Gastroenterology 109: 1612-1617, 1995.

35. Tannapfel A, Engeland K, Weinans L, Katalinic A, Hauss J, Mössner J and Wittekind C: Expression of p73, a novel protein related to the $\mathrm{p} 53$ tumour suppressor p53, and apoptosis in cholangiocellular carcinoma of the liver. Br J Cancer 80: 1069-1074, 1999. 
36. Teh M, Wee A and Raju GC: An immunohistochemical study of p53 protein in gallbladder and extrahepatic bile duct/ampullary carcinomas. Cancer 74: 1542-1545, 1994.

37. Washington $\mathrm{K}$ and Gottfried MR: Expression of $\mathrm{p} 53$ in adenocarcinoma of the gallbladder and bile ducts. Liver 16: 99-104, 1996.

38. Labib PL, Davidson BR, Sharma RA and Pereira SP: Locoregional therapies in cholangiocarcinoma. Hepat Oncol 4: 99-109, 2017.

39. Shinohara E, Mitra N, Guo M and Metz J: Radiation therapy is associated with improved survival in the adjuvant and definitive treatment of intrahepatic cholangiocarcinoma. Int J Radiat Oncol Biol Phys 72: 1495-1501, 2008.

40. Siebenhüner AR, Seifert H, Bachmann H, Seifert B, Winder T, Feilchenfeldt J, Breitenstein S, Clavien PA, Stupp R, Knuth A, et al: Adjuvant treatment of resectable biliary tract cancer with cisplatin plus gemcitabine: A prospective single center phase II study. BMC Cancer 18: 72, 2018.
41. Horgan AM, Amir E, Walter T and Knox JJ: Adjuvant therapy in the treatment of biliary tract cancer: A systematic review and meta-analysis. J Clin Oncol 30: 1934-1940, 2012

42. McNamara MG, Walter T, Horgan AM, Amir E, Cleary S, McKeever EL, Min T, Wallace E, Hedley D, Krzyzanowska M, et al: Outcome of adjuvant therapy in biliary tract cancers. Am J Clin Oncol 38: 382-387, 2015.

43. Stein A, Arnold D, Bridgewater J, Goldstein D, Jensen LH, Klümpen HJ, Lohse AW, Nashan B, Primrose J, Schrum S, et al: Adjuvant chemotherapy with gemcitabine and cisplatin compared to observation after curative intent resection of cholangiocarcinoma and muscle invasive gallbladder carcinoma (ACTICCA-1 trial)-a randomized, multidisciplinary, multinational phase III trial. BMC Cancer 15: 564, 2015. 